\title{
Vortex formation by unstable oceanic jets
}

\author{
Glenn R. Flierl ${ }^{1}$, Xavier J. Carton ${ }^{2}$ and Christophe Messager ${ }^{3}$ \\ (1) MIT/CMPO; Cambridge, MA 02139; USA \\ (2) EPSHOM/CMO; 29275 Brest; France; carton@shom.fr
}

(3) IFREMER/LPO; 29280 Plouzane; France

\begin{abstract}
In a two-layer quasi-geostrophic model, we investigate the mixed barotropic-baroclinic instability of a thin zonal jet. Given the jet velocity profile at the surface, the deformation radius and layer depths, the three parameters governing the instability are the ratio of layerwise maximum velocities $\left(U_{2} / U_{1}\right)$, the planetary vorticity gradient ( $\beta$-effect), and the wavenumber $(k)$ of the perturbation. First, simple criteria provide thresholds in this parameter space for the onset of instability. Growth rates of monochromatic perturbations are then computed and their most unstable wavelengths are compared with oceanic observations. With a nonlinear numerical model, high-Reynolds-number evolutions of the perturbed jet are computed in the $\left(U_{2} / U_{1}, \beta, k\right)$ space: wave breaking and vortex formation occur at small $\beta$, while for larger values of $\beta$, the perturbation equilibrates into meanders. Multiple waves are also observed. Instability is minimum when the jet is confined in the upper layer $\left(U_{2} / U_{1}=0\right)$. Finally, vortex formation by this unstable jet is specifically studied for a localized perturbation.
\end{abstract}

\section{Introduction}

The Gulf-Stream is a conspicuous feature of the north-western Atlantic ocean. This intense current can be idealized on part of its course as a thin jet, with a velocity profile strongly sheared, both horizontally and vertically. Thus it is sensitive to barotropic and baroclinic instabilities (Holland \& Haidvogel, 1980), and it meanders, with wavelengths ranging between 260 and $350 \mathrm{~km}$ (Kontoyannis \& Watts, 1994; Lee, 1994). These meanders, which amplify eastward, can undergo occlusion and form vortices. Then, these vortices detach from the jet, and drift westward. They are sometimes re-absorbed by the jet later.

Jet instability has already been studied in a two-dimensional flow (Flierl et al., 1987). Without $\beta$ effect (the planetary vorticity gradient), unstable thin jets form vortices. Conversely, only meanders develop on unstable jets for large $\beta$-effect. Here, jet instability is investigated in a two-layer flow, and the influence of several physical parameters (vertical shear of jet velocity $U_{2} / U_{1}$, $\beta$-effect, perturbation wavenumber $k$ ) on the jet evolution is quantified.

The model equations and stability criteria are presented in section 2. Section 3 is devoted to the study of growth rates of monochromatic, normal-mode perturbations on the jet, for various $\left(U_{2} / U_{1}, \beta, k\right)$. In section 4 , the nonlinear evolutions of the perturbed jet are investigated numerically in this parameter space, by means of a pseudo-spectral code. This study draws the limit between vortex formation and finite-amplitude wave stabilization. These processes are examined in section 5. Finally, in section 6, vortex formation is studied for a localized initial perturbation. 


\section{Model equations and stability criteria}

\subsection{Model equations}

The quasi-geostrophic equations govern flows strongly constrained by the Earth rotation and by the ocean stratification. In the inviscid and unforced limit, they express the conservation of potential plus planetary vorticities

$$
\frac{d\left(q_{j}+f\right)}{d t}=\partial_{t} q_{j}+J\left(\psi_{j}, q_{j}\right)+\beta \partial_{x} \psi_{j}=0 .
$$

For a two-layer flow, the upper,lower layer index is $j=1,2 ; J(a, b)=\partial_{x} a \partial_{y} b-\partial_{x} b \partial_{y} a$ is the Jacobian operator. The planetary vorticity is $f=f_{0}+\beta y$, with $f_{0}$ defined at the mid-latitude of the domain. The horizontal velocity is $u_{j}=\partial_{y} \psi_{j}, v_{j}=\partial_{x} \psi_{j}$. The potential vorticity is the sum of the curl of the velocity and of the vertical vortex stretching

$$
q_{j}=\nabla^{2} \psi_{j}+\gamma^{2} h_{k}\left(\psi_{k}-\psi_{j}\right), \quad k=3-j,
$$

where $h_{j}=H_{j} / H$ are scaled layer thicknesses and $\gamma=1 / R_{d}$ quantifies the layer coupling. The deformation radius $R_{d}$ is related to the Burger number by $B u=\left(R_{d} / W\right)^{2} \sim 1$, where $W$ is a horizontal scale of motion.

The model parameters are scaled on the Gulf-Stream, which flows in the upper 800 meters in a $4000 \mathrm{~m}$ deep ocean; thus $h_{1}=0.2, h_{2}=0.8$. The maximum jet velocity is $U_{1}=1.0 \mathrm{~m} . \mathrm{s}^{-1}$, here the velocity scale. For the Gulf-Stream, a half-width $W=35 \mathrm{~km}$ is commonly found (Watts, 1983). The deformation radius is $R_{d}=30 \mathrm{~km}$ at $35-40^{\circ} N, 45-65^{\circ} \mathrm{W}$ (Emery et al., 1984); $R_{d}$ is chosen here as the length scale. The time scale is $T=R_{d} / U_{1}=310^{4} \mathrm{~s}$. Finally, the planetary vorticity gradient is $\beta \sim 1.7410^{-11} \mathrm{~m}^{-1} \mathrm{~s}^{-1}$ at $40^{\circ} \mathrm{N}$; it is normalized as $\beta^{*}=\beta R_{d}^{2} / U_{1}$.

We decompose the flow into a stationary, zonal jet (with weak lateral countercurrents) and a time-dependent normal-mode perturbation:

$$
\begin{gathered}
\psi_{j}(x, y, t)=\bar{\psi}_{j}(y)+\psi_{j}^{\prime}(x, y, t), \\
\bar{\psi}_{j}(y)=-U_{j} w\left[\tanh (y / w)-\frac{y}{L_{b}} \tanh \left(\frac{L_{b}}{w}\right)\right], \quad U_{2}=\alpha U_{1} \\
\psi_{j}^{\prime}(x, y, t)=\phi_{j}(y) e^{i k(x-c t)} .
\end{gathered}
$$

The size of the numerical model domain is $L_{b} ; w=W / R_{d}$ is the scaled jet half-width; $\alpha=U_{2} / U_{1}$ characterizes the vertical structure of jet velocity. With $c=c_{r}+i c_{i}$, the growth rate of the perturbation is $\sigma=k c_{i}$. The potential vorticity equation is linearized around the mean flow to form the Rayleigh equation for linear instability:

$$
\left(\bar{u}_{j}-c\right) q_{j}^{\prime}+\left(\beta^{*}+\frac{d \bar{q}_{j}}{d y}\right) \psi_{j}^{\prime}=0, \text { for } j=1,2
$$

which is a generalized eigenvalue problem solved by a matrix method. It has been checked that the growth rates are nearly insensitive to the domain size, provided $L_{b}>>w$ (see also Flierl et al., ibid). 


\section{$2.2 \quad$ Stability criteria}

Two instabilities can affect quasi-geostrophic jets: the barotropic one is due to strong horizontal shears of vorticity and is associated with kinetic energy transfer from the mean flow to the perturbation; the baroclinic one is due to the tilting of isopycnal surfaces over isobars; it is associated with the release of potential energy of the mean flow to eddy kinetic energy. Criteria provide qualitative thresholds for the halting of barotropic and of baroclinic instabilities by $\beta$-effect.

The Kuo criterion for barotropic instability (Pedlosky, 1987) states that $\beta^{*}+\frac{d \overline{q_{t}}}{d y}$ must change sign for perturbations to grow; $\overline{q_{t}}=h_{1} \overline{q_{1}}+h_{2} \overline{q_{2}}$ is the mean barotropic potential vorticity. Here,

$$
\frac{d \bar{q}_{t}}{d y}=2 U_{1}\left(h_{1}+\alpha h_{2}\right)\left[1-2 \sinh ^{2}(y / w)\right] /\left[w^{2} \cosh ^{4}(y / w)\right]
$$

with $\alpha=1$ and $h_{1}+h_{2}=1$. This vorticity gradient has a minimum negative value for $\sinh (y / w)=$ $\sqrt{2}$. The critical value of the $\beta$-effect which restores stability of a barotropic jet is then $\beta_{c}^{*}=$ $2 U_{1} / 3 w^{2}$ (here $\beta_{c}^{*}=0.5$ ).

For baroclinic instability in quasi-geostrophic flows, the Charney-Stern theorem (Pedlosky, 1987) states that $d \overline{q_{j}} / d y+\beta^{*}$ must change sign in or between layers for instability to develop. The critical value of $\beta^{*}$ which stabilizes the jet decreases from $\beta_{c}^{*}=1.87$ at $\alpha=-1.0$ to $\beta_{c}^{*}=0.28$ at $\alpha=0.0$ and increases back to $\beta_{c}^{*}=0.5$ at $\alpha=1.0$. Surface-confined jets are thus the least unstable ones.

A wavenumber-dependent stability criterion can be obtained by using the two-layer Phillips model (Pedlosky, 1987), for a horizontally uniform, and vertically sheared flow. The necessary condition for instability is then:

$$
\Delta=\beta^{* 2} \gamma^{4}+2 \beta^{*}\left(U_{1}-U_{2}\right) \gamma^{2}\left(h_{2}-h_{1}\right) k^{4}-k^{4}\left(U_{1}-U_{2}\right)^{2}\left(4 \gamma^{4} h_{1} h_{2}-k^{4}\right)<0
$$

Here, we set $U_{1}=U_{1 \max }, U_{2}=U_{2 \max }$ to obtain a reasonable approximation of the critical value $\beta^{*}$ which stabilizes the jet:

$$
\beta_{c}^{*}=\frac{U_{1}(1-\alpha) k^{4}}{\gamma^{2}}\left[h_{2}-h_{1}+2 \sqrt{h_{1} h_{2}} \sqrt{\gamma^{4} / k^{4}-1}\right]
$$

for $k<\gamma$ or $\lambda>2 \pi R_{d}$ (here $188.5 \mathrm{~km}$ ). The critical values for stability are $\beta_{c}^{*}=0.8,1.6$ when $\alpha=0.0,-1.0$ at $k=0.95$.

\section{Linear jet stability; growth rates of monochromatic perturba- tions}

The Rayleigh equation is first solved in the two-dimensional case $(\gamma=0)$ as a reference. The instability vanishes for $\beta^{*}=0.5$ (Fig. 1a), the value given by the Kuo criterion. The maximum growth rate shifts to smaller wavelengths with increasing $\beta^{*}: k$ goes from 0.80 to 1.20 ( $\lambda$ shifts from 235 to $160 \mathrm{~km}$ ) when $\beta^{*}$ increases from zero to 0.5 .

Next, the Rayleigh equation is solved for the two-layer jet. The results for $\alpha=1.0$ are identical to those of the two-dimensional case and are thus not shown. Fig. 1b (resp. 1c) presents the growth rates in the $\left(\beta^{*}, k\right)$-plane, for $\alpha=0.0$ (resp. -1.0). We restrict our study to sinuous perturbations which are much more unstable than varicose waves (as in Flierl et al., 1987). The intensity of baroclinic instability is related to $\alpha$ : the growth rate $\sigma$ vanishes for $\beta_{c}^{*} \sim 0.3,1.9$, when 


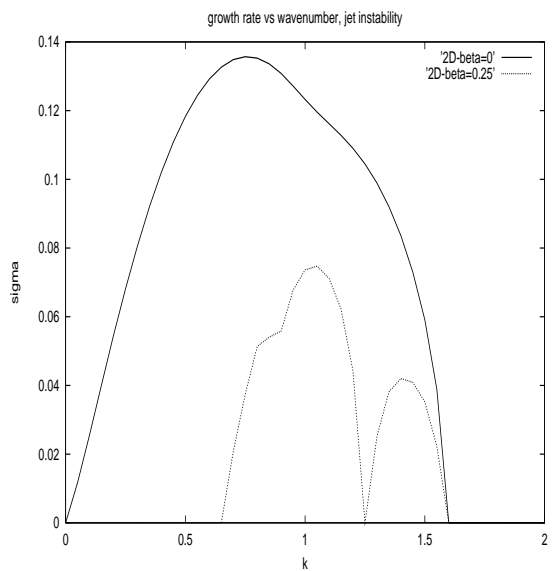

(a)

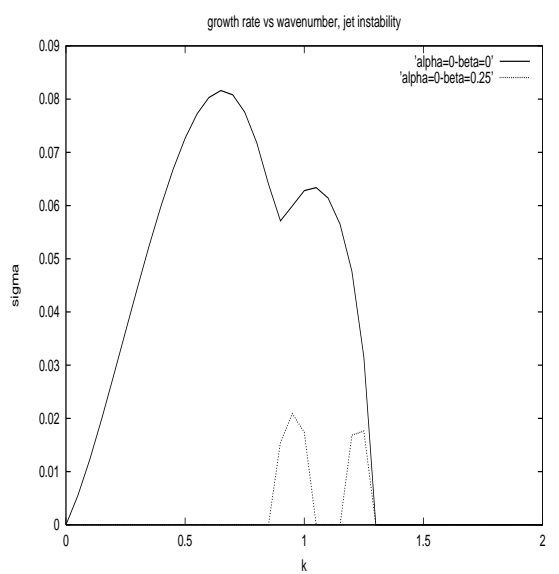

(b)

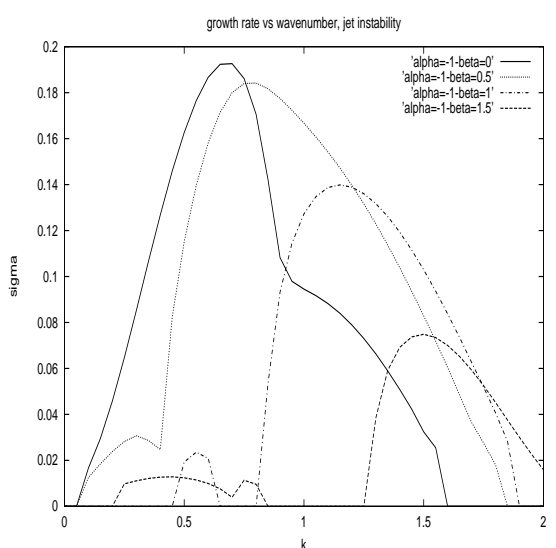

(c)

Figure 1: Linear instability of the zonal jet of section (2.1); (a) Growth rates versus k, for $\alpha=1.0$ and various values of $\beta^{*}$; (b) same as (1a) for $\alpha=0.0$; (c) same as (1a) for $\alpha=-1.0$. 
$\alpha=0.0,-1.0$. These values are identical to those given by the Charney-Stern criterion.

For $\alpha=0.0$ : at $\beta^{*}=0$, long waves $(k=0.65, \lambda=290 \mathrm{~km})$ are unstable, but with much smaller growth rates than for both $\alpha=-1.0$ and $\alpha=1.0(\sigma=0.082)$; at $\beta^{*}=0.2$, long waves are unstable $(k=0.55-0.95, \lambda=200-350 \mathrm{~km}, \sigma=0.03)$.

For $\alpha=-1.0$ : at $\beta^{*}=0$, long waves $(k=0.65, \lambda=290 \mathrm{~km})$ are the most unstable $(\sigma=0.19)$. At $\beta^{*}=1.5$, short waves $(k=1.45, \lambda=130 \mathrm{~km})$ are dominant $(\sigma=0.075)$, while long waves are marginally unstable.

In all cases, the unstable long waves are predicted by the Phillips model.

With the eigenvectors of the linear stability problem

$$
\phi_{j}(y)=A_{j}(y) e^{i \theta_{j}(y)}
$$

the kinetic and potential energy transfers between the mean jet and the perturbation can be computed as

$$
\begin{gathered}
T K_{j}=k h_{j} \int A_{j}^{2} \frac{d \theta_{j}}{d y} \frac{d \bar{u}_{j}}{d y} d y \\
T P=\frac{1}{2} k \gamma^{2} h_{1} h_{2} \int\left(\bar{u}_{2}-\bar{u}_{1}\right) A_{1} A_{2} \sin \left(\theta_{1}-\theta_{2}\right) d y .
\end{gathered}
$$

For $\alpha=0, \beta^{*}=0$, calculations show that upper layer kinetic energy transfer has an amplitude similar to that of the potential energy transfer, but for shorter waves. The two peaks in the growth rate diagram are explained by the successive maxima of potential and of kinetic energy transfers when $k$ increases.

In summary:

- the agreement between the simple stability criteria and the linear stability analysis is good: the values of $\beta^{*}$ stabilizing the jet are similar; the Phillips model predicts the unstable long waves;

- the values of $\beta^{*}$ stabilizing the jet are much larger than the dimensionless value of the Earth vorticity gradient $\beta_{g}^{*}=1.6710^{-2}$. The Gulf-Stream is thus linearly unstable;

- at $\beta^{*}=0$, the most unstable wavelengths $(\lambda \in[235,290 \mathrm{~km}])$ vary little with the vertical structure of the jet, $\alpha$;

- with increasing $\beta^{*}$, the most unstable wavelengths remain close to Gulf-Stream observations only for $\alpha=0.0$; then, the growth periods vary from 4 days with $\beta^{*}=0$ to 16 days with $\beta^{*}=0.2$. This is comparable with the 3-15 days observed periods (Kontoyannis \& Watts, 1994; Lee, 1994);

- increasing $\beta^{*}$ stabilizes long waves;

- baroclinic instability is slightly stronger than the barotropic one at $\alpha=0.0$ (as for the GulfStream), but much stronger at $\alpha=-1.0$. The respective strengths of barotropic and baroclinic instabilities are quantified by the computation of energy transfers.

\section{Classification of nonlinear regimes}

The nonlinear quasi-geostrophic equations are now solved numerically by means of a pseudospectral code, with the zonal jet and a weak normal-mode perturbation as initial conditions. The equations are discretized on a biperiodic $(\mathrm{x}, \mathrm{y})$ grid. The box size is $L_{b}=8 \pi(754 \mathrm{~km})$ with 128 nodes in each direction in section 4 ; it is $L_{b}=16 \pi(1508 \mathrm{~km})$ with 256 nodes in section 5. Spatial derivatives are performed via Fourier-Galerkin transforms, and the time advection is a mixed Euler-leapfrog scheme. The Courant-Friedrich-Lewy stability condition is met. In the spectral code, the right-hand side of Eq. 1 is replaced by a biharmonic friction $\left(\nu \nabla^{6} \psi_{j}\right)$, with a 
small coefficient $\left(\nu=10^{-7}\right)$. This numerical dissipation prevents enstrophy accumulation at small scales. Total energy decays by less than $1 \%$ (and total enstrophy by less than $2 \%$ ) over a 180-day model run. Roughly 200 numerical simulations have been performed with the quasi-geostrophic model to map the finite-amplitude evolutions of the unstable jet in the $\left(\alpha, \beta^{*}, k\right)$ space.

Firstly, for a surface-confined jet $(\alpha=0.0)$, the finite-amplitude behaviors in the $\left(\beta^{*}, k\right)$ plane are summarized in Table 1 . For $\beta^{*}=0$, long waves amplify by nonlinear interactions, break and form eddies; shorter waves equilibrate into finite amplitude meanders. At small $\beta^{*}$, the dominant wavenumber evolves to $k=0.75$, the linearly most unstable. For larger $\beta^{*}$, larger wavenumbers are more unstable, since $\beta$-effect stabilizes long waves.

Secondly, for $k=0.25,0.75,1.25$, the finite-amplitude behaviors in the $\left(\alpha, \beta^{*}\right)$ plane are summarized in Tables 2a, b, c. The surface-confined jet is slightly less unstable than the barotropic jet $(\alpha=1.0)$ and much less than the fully baroclinic jet $\alpha=-1.0$, in agreement with the linear analysis. So, baroclinic instability is more efficient than barotropic instability at creating eddies. In general, for $\beta^{*}=0$, only long waves break. When $\beta^{*}$ increases, mode $k=0.75$ finally dominates shorter or longer waves. Still, when $\beta^{*}$ is quite large $\left(\beta^{*}=0.4\right.$ to 1.5$)$, short waves can be produced $(k=1$ or $k=1.25)$, again in agreement with the stabilizing effect of $\beta$ on long waves; in some instances, multiple waves co-exist.

Comparing surface-confined jets $(\alpha=0.0)$ jets with two-dimensional ones (Flierl et al., 1987), we observe that fewer dipoles are formed in the former case. For $\beta^{*}=\beta_{g}^{*}$ (the Earth vorticity gradient), surface-confined jets form vortices by direct amplification of waves, while for $\beta^{*}=2 \beta_{g}^{*}$, vortices are formed after wavenumber bifurcations and interactions between multiple waves.

We finally note that the critical values of $\beta^{*}$ which completely stabilize the jets are similar in the nonlinear experiments and in linear stability theory.

In summary, the nonlinear evolutions confirm and complement the previous stability analysis:

- the most unstable wave amplifies the fastest; if it is not initialized explicitly, it grows from numerical noise. Bifurcations from the initial perturbation towards this most unstable wave occur after a finite time;

- the surface-intensified jet (e.g. the Gulf-Stream) is more stable than the barotropic or fully baroclinic one; in general, baroclinic instability is more powerful than barotropic instability for vortex production;

- vortex formation stops and waves stabilize at finite amplitude for values of $\beta^{*}$ always smaller than 0.2 whatever $\alpha$;

- vortices can be formed by the interaction of multiple waves, even if the dominant one alone does not form vortices;

- beta effect stabilizes long waves; its critical value for complete jet stability in the nonlinear model is close to the linear $\beta_{c}^{*}$.

\section{Analysis of nonlinear regimes}

\subsection{Vortex generation and detachment}

We investigate vortex formation by an unstable, surface-confined jet $(\alpha=0.0)$, perturbed on wavenumber $k=0.75$ and with $\beta^{*}=0$. Fig.2a shows the upper and lower layer potential vorticities and the vertical deviation of the interface between layers, $\eta \propto \psi_{2}-\psi_{1}$. Until 28 days, the meanders amplify without much deformation. Later, they present a strong phase shift, both meridional and 
vertical, opposed to the mean velocity shear. Such a phase shift is a sufficient condition for barotropic and baroclinic instabilities (Pedlosky, 1987). During the vortex detachment stage (56 days), this phase shift results in a northeastward direction of the ejected vortices, and in their coupling as two-layer vortex pairs (see $q_{1}$ and $q_{2}$ at 56 days).

The interface deviation is vertically aligned with the upper-layer potential vorticity front, which is dynamically dominant over the lower-layer one. In the strongly curved meanders, the streamwise geostrophic acceleration is balanced by a spanwise ageostrophic Coriolis effect. This ageostrophic velocity induces successive streamline convergence and divergence, corresponding respectively to upwards and downwards motions of the interface.

Finally, the modal analysis of the nonlinear evolution is shown in Fig.2b. To perform it, the zonally averaged jet is substracted from the total flow at each instant. Until 32 days, the exponential growth of the fundamental wave $k=0.75$ follows the linear instability prediction. The second growing wave (after 16 days) is the first harmonic $k=1.5$, which results from the self interaction of the $k=0.75$ wave. After 38 days, a second stage begins: long waves $(k=0.25,0.5)$ amplify, feeding on the fundamental wave whose energy is depleted. Vortices start detaching (near 56 days) when very long waves amplify to finally dominate.

\subsection{Finite-amplitude equilibration of meanders}

To illustrate the finite-amplitude equilibration of meanders, we study a surface-confined jet $(\alpha=$ 0.0 ), perturbed on wavenumber $k=0.5$ and with $\beta^{*}=0.05$. Fig.3a shows the layerwise potential vorticities and the interface deviation, during the stage of nonlinear stabilization. We note that the front separating negative and positive potential vorticities is vertically aligned between the two layers. The interface deviation is also vertically aligned with the upper-layer meanders. This shows that the linear instability has ceased. Meander steepening, shown here between days 63 and 70 , is due to shorter waves. In fact, oscillations between smooth and steep meanders last until the end of the simulation.

The modal analysis of this simulation is shown in Fig.3b. After the initial exponential growth of mode $k=0.5$, an amplitude decrease leading to stabilization is observed. Contrary to the case of vortex formation, the first harmonic $k=1.0$ grows rapidly and significantly, followed by the second harmonic $k=1.5$. A second noticeable difference is the very weak amplitude of the other waves. Thus long waves are necessary for vortex detachment.

\subsection{Wavenumber bifurcations and vortex formation}

Finally, we present an example of wave transition (bifurcation between wavenumbers) with $\alpha=0$, $k=1.25, \beta^{*}=0.0323$. Fig.4a shows that after two months, the fundamental wave has not grown into significant meanders. A slow transition towards wavenumber $k=0.75$ is clear after 133 days. Steepening of the meanders follow and their interaction leads to vortex formation and to long waves $(k=1)$. The process of vortex formation is particularly explicit in the upperlayer potential vorticity and interface deviation plots: the westernmost southward meander in undergoing occlusion, while the next one east is still open. Farther east, a vortex has already detached and is propagating along the jet. The modal analysis (Fig.4b) confirms that wavenumber $k=1.25$ is barely amplifying. The other waves grow from numerical noise and via nonlinear interactions. Mode $k=0.75$ is the fastest-growing wave as predicted by the linear theory. Again eddy detachment occurs when many waves have reached a large amplitude (around 150 days). 

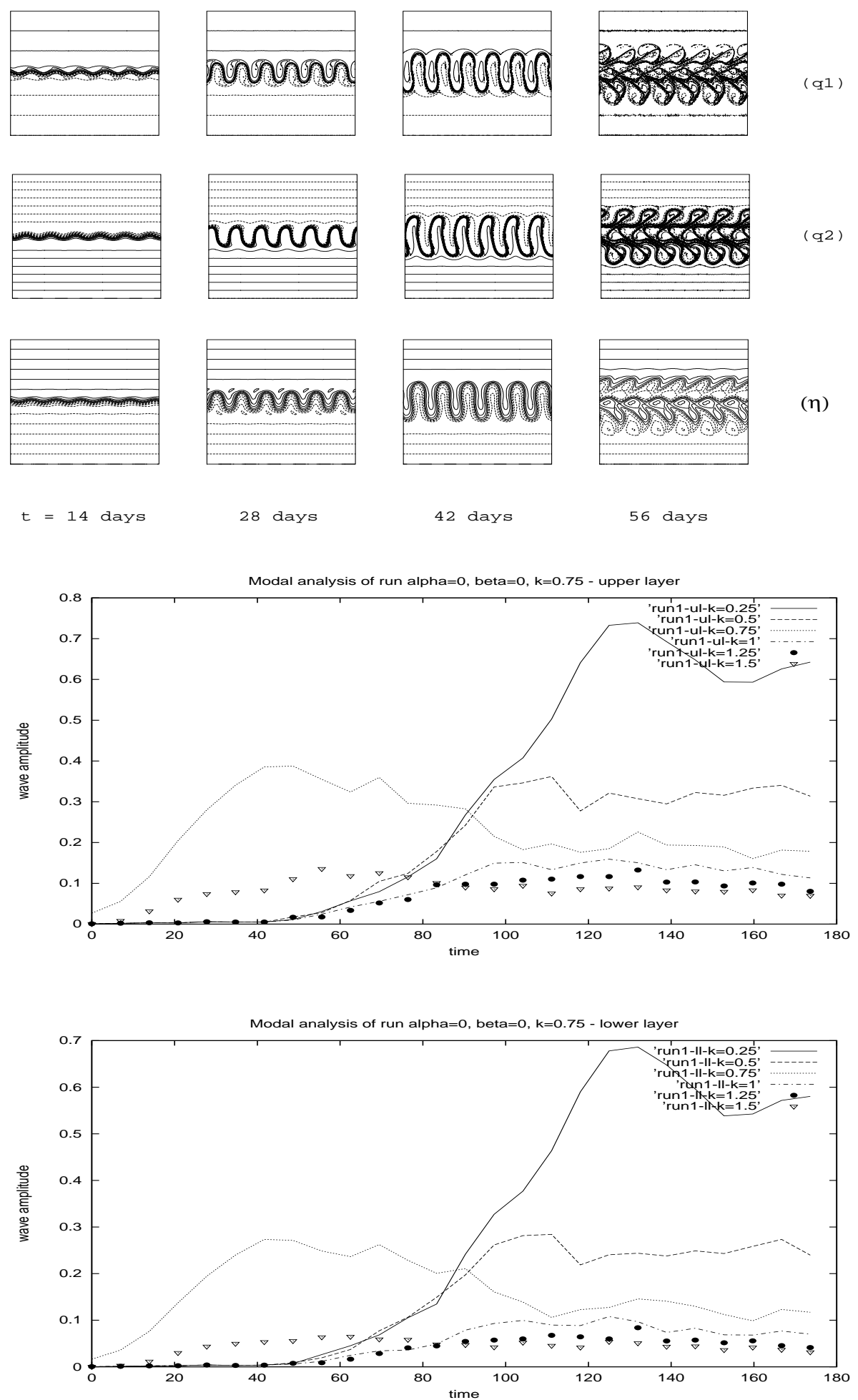

Figure 2: Vortex formation by an unstable jet - (a) upper and lower potential vorticities and interface deviation of the $\alpha=0$ jet, perturbed with a $k=0.75$ sinusoidal wave, with $\beta=0.0$; (b) modal analysis of this nonlinear evolution: $k=0.25, \ldots, 1.5$ wave amplitudes versus time. 

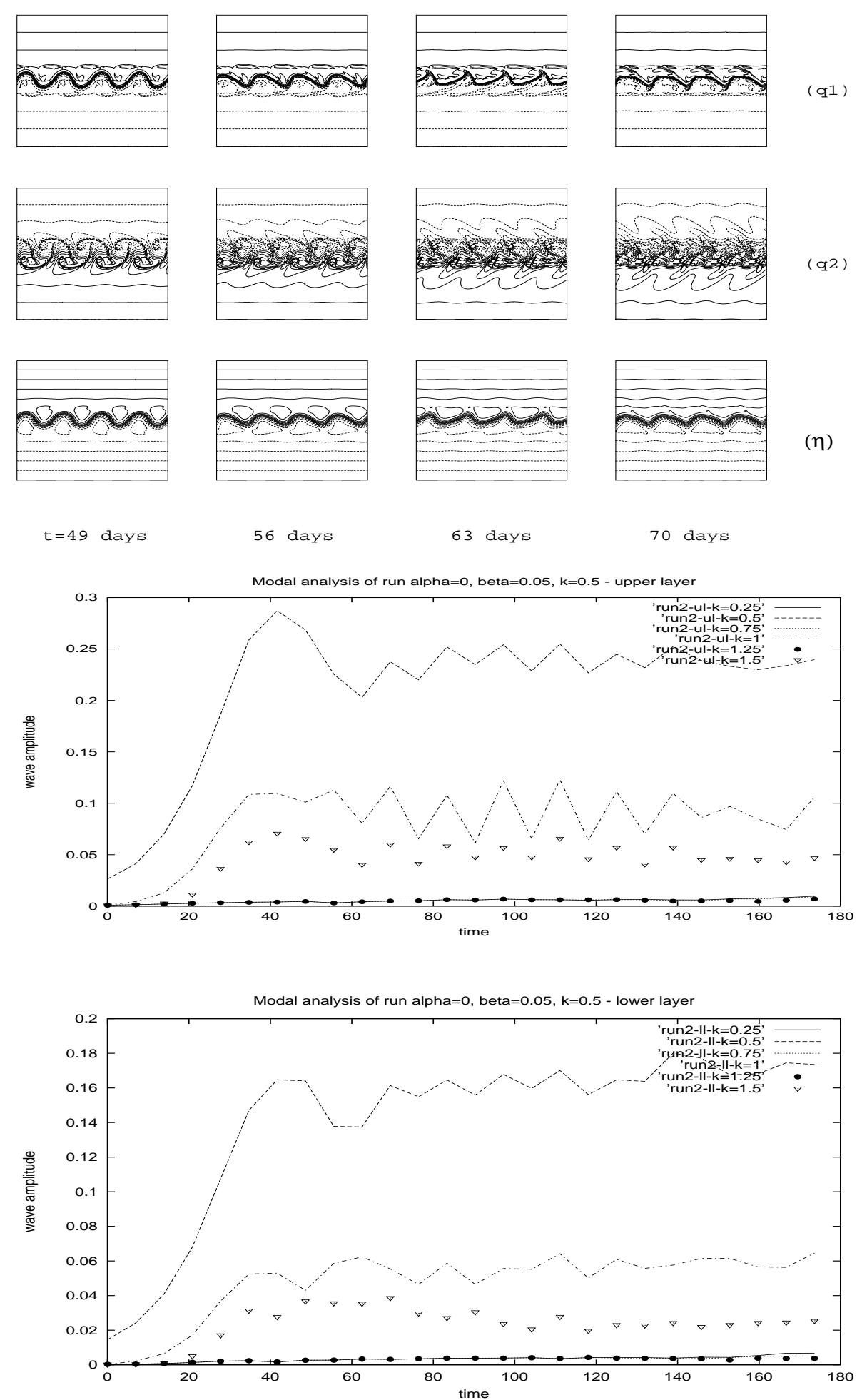

Figure 3: Finite-amplitude meander stabilization - (a) upper and lower potential vorticities and interface deviation of the $\alpha=0$ jet, perturbed with a $k=0.5$ sinusoidal wave, with $\beta=0.05$; (b) modal analysis of this nonlinear evolution: $k=0.25, \ldots, 1.5$ wave amplitudes versus time. 

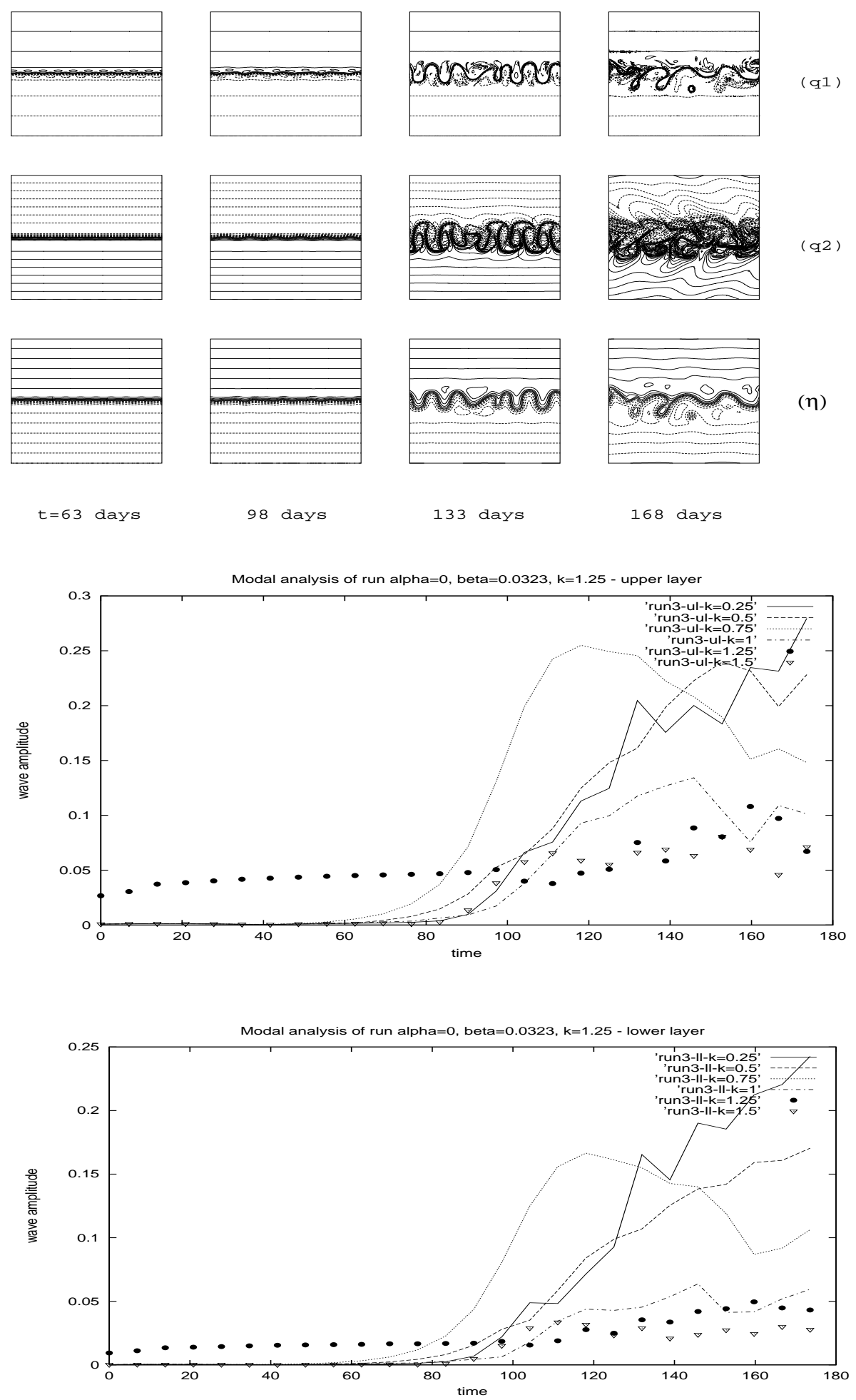

Figure 4: Bifurcations between waves - (a) upper and lower potential vorticities and interface deviation of the $\alpha=0$ jet, perturbed with a $k=1.25$ sinusoidal wave, with $\beta=0.0323$; (b) modal analysis of this nonlinear evolution: $k=0.25, \ldots, 1.5$ wave amplitudes versus time. 


\section{Vortex formation from localized perturbations on the jet}

In this section, we add to the jet a more realistic perturbation than a sinusoidal wave: a Gaussian northward deviation of the potential vorticity isolines idealizes a local disturbance. The northward displacement is $5 \mathrm{~km}$ and the zonal e-folding scale is $15 \mathrm{~km}$. The spectral code is run with $L_{b}=8 \pi$ and 128 nodes in each direction, to determine the nonlinear evolutions of this jet. The cases $\alpha=0.0,-1.0$ and $\beta \in[0.0,0.15]$ are considered.

For $\alpha=0.0$, the most unstable wave $k=0.75$ amplifies and sheds vortices for $\beta^{*} \leq 0.05$ (see Table 3 for the number of vortices formed). For $\beta^{*}>0.05$, the $k=0.75$ wave stabilizes at finiteamplitude. No specific asymmetry between cyclones and anticyclones is observed (neither in size, nor in number).

For $\alpha=-1.0$, the most unstable wave $k=0.75$ amplifies and sheds vortices for $\beta^{*} \leq 0.1$. For small $\beta^{*}$, vortex dipoles are formed and ejected on both sides of the jet. For $\beta^{*}>0.1$, meanders equilibrate. Again, no specific asymmetry is observed between cyclones and anticyclones.

\section{Conclusions}

In a quasi-geostrophic model, the instability of a thin zonal jet has been investigated with respect to three parameters: the vertical shear of velocity, the planetary vorticity gradient, the wavenumber of the perturbation. The physical results are the following:

- baroclinic instability is generally stronger than barotropic instability. Both have similar strengths in the case of the surface-confined jet, the least unstable one. Without $\beta$-effect, the most unstable wavelengths are those of Gulf-Stream meanders. Increasing $\beta^{*}$ stabilizes long waves, but all wavelengths are linearly stabilized only for values of $\beta^{*}$ much larger than the Earth vorticity gradient. - For a surface-confined jet, nonlinear simulations show that vortex formation ceases for values of $\beta^{*}$ comparable with the Earth planetary vorticity gradient. The linearly most unstable wave grows the fastest, even from numerical noise if it is not initialized explicitly.

- The modal analysis of nonlinear simulations reveals that vortex detachment is correlated with the growth of long waves, while meander stabilization occurs when only the fundamental wave and its first harmonics (short waves) are present. Since beta-effect stabilizes long waves first, this explains why only a low value of $\beta^{*}$ suffices to prevent vortex formation.

- When the jet is initially perturbed locally in physical space, the most unstable wave $k=0.75$ grows and vortices are shed. No asymmetry is found between vortices of opposite polarities.

Finally, this study is extended to ageostrophic dynamics (in a shallow-water model) in the companion paper (Baey et al., 1998).

\section{Ackowledgements}

Funding for this study was provided by the Massachussets Institute of Technology (USA), and by the Service Hydrographique et Océanographique de la Marine (France). The second author gratefully acknowledges Pr Sadourny's hospitality at LMD (Paris) and discussions with Dr C. Provost (LODYC, Paris).

\section{References}

1 Baey J.M., Riviere P. And X. Carton: Ocean jet instability: a model comparison. Proceedings of the 3rd IWVF, this volume (1998) 
2 Emery W.J., Lee W.G. And L. MagaArd: Geographic and seasonal distributions of Brunt-Vaisala frequency and Rossby radii in the North Pacific and North Atlantic. J. Phys. Oceanogr. 14 (1984), $294-317$.

3 Flierl G.R., Malanote-Rizzoli P. and N.J. Zabusky: Nonlinear waves and coherent vortex structures in barotropic $\beta$-plane jets. J. Phys. Oceanogr. 17 (1987), 1408-1438.

4 Holland W.R. And D.B. Haidvogel: A parameter study of the mixed instability of idealized ocean currents. Dyn. Atmos. Oceans, 4 (1980), 185-215.

5 Kontoyannis H. And D.R. WATts: Observations on the variability of the Gulf-Stream path between $74^{\circ} W$ and $70^{\circ} W$. J. Phys. Oceanogr. 24 (1994), 1999-2013.

6 LEE T.: Variability of the Gulf-Stream path as observed from satellite infrared images. PhD dissertation, University of Rhode Island (1994), 188 pp.

7 Pedlosky J.: Geophysical Fluid Dynamics. Springer-Verlag, 2nd ed. (1987), 710pp.

8 Watts, R.: "Gulf-Stream variability". In "Eddies in Marine Sciences" (Ed. A.R. Robinson), SpringerVerlag (1983), 114-143. 


\section{Tables}

\begin{tabular}{|c|c|c|c|c|c|}
\hline$\beta^{*} \backslash \mathrm{k}$ & 0.25 & 0.5 & 0.75 & 1.0 & 1.25 \\
\hline 0.0 & $\mathrm{VF}$ & $\mathrm{VF}$ & $\mathrm{VF}+\mathrm{DP}$ & $\mathrm{B}(3), \mathrm{VF}$ & $\mathrm{B}(3), \mathrm{VF}$ \\
\hline 0.016 & $\mathrm{~B}(3), \mathrm{VF}$ & $\mathrm{VF}$ & $\mathrm{FA}, \mathrm{VF}$ & $\mathrm{B}(3), \mathrm{VF}$ & $\mathrm{B}(3), \mathrm{VF}$ \\
\hline 0.032 & $\mathrm{~B}(3), \mathrm{VF}$ & $\mathrm{FA}$ & $\mathrm{FA}$ & $\mathrm{B}(3), \mathrm{VF}$ & $\mathrm{B}(3), \mathrm{VF}$ \\
\hline 0.05 & $\mathrm{~B}(3)$ & $\mathrm{FA}, \mathrm{R}$ & $\mathrm{FA}$ & $\mathrm{B}(3)$ & $\mathrm{B}(3)$ \\
\hline 0.1 & $\mathrm{~B}(3)$ & $\mathrm{SA}, \mathrm{MW}$ & $\mathrm{FA}$ & $\mathrm{B}(3)$ & $\mathrm{B}(3)$ \\
\hline 0.2 & $\mathrm{~S}$ & $\mathrm{~S}$ & $\mathrm{SA}$ & $\mathrm{SA}$ & $\mathrm{SA}$ \\
\hline 0.3 & $\mathrm{~S}$ & $\mathrm{~S}$ & $\mathrm{~S}$ & $\mathrm{~S}$ & $\mathrm{~S}$ \\
\hline
\end{tabular}

Table 1: Finite-amplitude regimes of a weakly perturbed continuous jet with $\alpha=0$, as a function of $k$ and $\beta^{*}$. The symbols are:

$\mathrm{B}(\mathrm{N})=$ bifurcation towards wavenumber $k=N * 0.25$,

$\mathrm{FA}=$ finite-amplitude meanders on the given wavenumber,

$\mathrm{MW}=$ final state with multiple waves,

$\mathrm{R}=$ resonance between square and sawtooth waves,

$\mathrm{S}=$ stable on the given wavenumber,

$\mathrm{SA}=$ small-amplitude meanders,

$\mathrm{VF}=$ vortex formation from wave breaking

$\mathrm{DP}=$ dipole formation.

\begin{tabular}{|c|c|c|c|c|c|c|}
\hline$\beta^{*} \backslash \alpha$ & -1.0 & -0.5 & -0.25 & 0.0 & 0.5 & 1.0 \\
\hline 0.0 & $\mathrm{VF}$ & $\mathrm{VF}$ & $\mathrm{VF}$ & $\mathrm{VF}$ & $\mathrm{VF}$ & $\mathrm{VF}$ \\
\hline 0.2 & $\mathrm{~B}(3)$ & $\mathrm{SA}, \mathrm{MW}$ & $\mathrm{B}(3)$ & $\mathrm{S}$ & $\mathrm{SA}$ & $\mathrm{B}(3)$ \\
\hline 0.4 & $\mathrm{~B}(4)$ & $\mathrm{SA}, \mathrm{MW}$ & $\mathrm{S}$ & $/ /$ & $\mathrm{S}$ & $\mathrm{S}$ \\
\hline 0.6 & $\mathrm{~B}(4)$ & $\mathrm{S}$ & $/ /$ & $/ /$ & $/ /$ & $/ /$ \\
\hline 0.8 & $\mathrm{~B}(3)$ & $/ /$ & $/ /$ & $/ /$ & $/ /$ & $/ /$ \\
\hline 1.0 & $\mathrm{~S}$ & $/ /$ & $/ /$ & $/ /$ & $/ /$ & $/ /$ \\
\hline
\end{tabular}

Table 2a: Finite-amplitude regimes of a weakly perturbed continuous jet, for a wavenumber $k=0.25$. Symbols are as in Table $1,(/ /)$ meaning no numerical simulation for this particular case.

\begin{tabular}{|c|c|c|c|c|c|c|}
\hline$\beta^{*} \backslash \alpha$ & -1.0 & -0.5 & -0.25 & 0.0 & 0.5 & 1.0 \\
\hline 0.0 & VF & VF & VF & VF & VF & VF \\
\hline 0.2 & FA & FA & FA & SA & SA & SA \\
\hline 0.4 & FA & FA & FA & S & S & S \\
\hline 0.6 & FA & SA & S & $/ /$ & $/ /$ & $/ /$ \\
\hline 0.8 & FA & S & $/ /$ & $/ /$ & $/ /$ & $/ /$ \\
\hline 1.0 & SA & $/ /$ & $/ /$ & $/ /$ & $/ /$ & $/ /$ \\
\hline 1.2 & SA & $/ /$ & $/ /$ & $/ /$ & $/ /$ & $/ /$ \\
\hline 1.5 & $\mathrm{~B}(5)$ & $/ /$ & $/ /$ & $/ /$ & $/ /$ & $/ /$ \\
\hline
\end{tabular}


Table 2b: Finite-amplitude regimes of a weakly perturbed continuous jet, for a wavenumber $k=0.75$. Symbols are as in Table $1,(/ /)$ meaning no numerical simulation for this particular case.

\begin{tabular}{|c|c|c|c|c|c|c|}
\hline$\beta^{*} \backslash \alpha$ & -1.0 & -0.5 & -0.25 & 0.0 & 0.5 & 1.0 \\
\hline 0.0 & $\mathrm{~B}(3)$ & $\mathrm{B}(3)$ & $\mathrm{B}(3)$ & $\mathrm{B}(3)$ & $\mathrm{B}(3)$ & $\mathrm{VF}$ \\
\hline 0.2 & $\mathrm{~B}(3)$ & $\mathrm{SA}$ & $\mathrm{SA}$ & $\mathrm{SA}$ & $\mathrm{S}$ & $\mathrm{SA}, \mathrm{B}(3)$ \\
\hline 0.4 & $\mathrm{SA}, \mathrm{MW}$ & $\mathrm{S}$ & $\mathrm{S}$ & $\mathrm{S}$ & $/ /$ & $\mathrm{S}$ \\
\hline 0.6 & $\mathrm{SA}, \mathrm{MW}$ & $/ /$ & $/ /$ & $/ /$ & $/ /$ & $/ /$ \\
\hline 0.8 & $\mathrm{SA}$ & $/ /$ & $/ /$ & $/ /$ & $/ /$ & $/ /$ \\
\hline 1.0 & $\mathrm{SA}$ & $/ /$ & $/ /$ & $/ /$ & $/ /$ & $/ /$ \\
\hline 1.2 & $\mathrm{~S}$ & $/ /$ & $/ /$ & $/ /$ & $/ /$ & $/ /$ \\
\hline
\end{tabular}

Table 2c: Finite-amplitude regimes of a weakly perturbed continuous jet, for a wavenumber $k=1.25$. Symbols are as in Table $1,(/ /)$ meaning no numerical simulation for this particular case.

\begin{tabular}{|c|c|c|c|c|c|c|c|c|}
\hline$\alpha \backslash \beta^{*}$ & 0.0 & 0.01 & 0.0167 & 0.025 & 0.0323 & 0.05 & 0.1 & 0.15 \\
\hline 0.0 & $1 \mathrm{C}, 1 \mathrm{AC}$ & $1 \mathrm{C}, 1 \mathrm{AC}$ & $1 \mathrm{C}, 1 \mathrm{AC}$ & $1 \mathrm{C}$ & $1 \mathrm{C}, 2 \mathrm{AC}$ & $1 \mathrm{AC}$ & 0 & 0 \\
\hline-1.0 & $4 \mathrm{DP}$ & $2 \mathrm{DP}$ & $2 \mathrm{DP}$ & $1 \mathrm{DP}$ & $1 \mathrm{C}$ & $1 \mathrm{C}$ & $1 \mathrm{C}$ & 0 \\
\hline
\end{tabular}

Table 3: Number of cyclones (C), anticyclones (AC) or dipoles (DP) formed by an unstable jet with $\alpha, \beta^{*}$ given hereabove, with a localized perturbation. 